\title{
Imaging protein kinase A signaling dynamics in live cardiac myocytes
}

\author{
J.J. Saucerman, ${ }^{*}$ J. Zhang, ${ }^{* *}$ A.E. Stenbit, ${ }^{* * *}$ R.Y. Tsien****, and A.D. McCulloch* \\ * Department of Bioengineering, Whitaker Institute of Biomedical Engineering, University of \\ California, San Diego, La Jolla, CA 92093 \\ ** Department of Pharmacology, Johns Hopkins School of Medicine, Baltimore, MD 21205 \\ *** School of Medicine, University of California, San Diego, La Jolla, CA 92093 \\ **** Departments of Pharmacology and Chemistry and Biochemistry, Howard Hughes Medical \\ Institute, University of California, San Diego, La Jolla, CA 92093
}

Protein kinase A (PKA) signaling helps to coordinate the sympathetic response of the heart, important to both normal function and the development of disease. While a number of cardiac signaling pathways act through PKA, many of these lead to distinct physiologic responses. Such observations led to the hypothesis that cyclic AMP/PKA signaling is highly localized [1]. While traditional means of measuring cell signaling require destruction of the cell, averaging over large populations, or have poor spatiotemporal resolution, genetically-encoded FRET biosensors provide an opportunity to image signaling dynamics in single living cells. These fluorescent biosensors may be useful for elucidating the role of dynamics and compartmentation in controlling the physiologic response to PKA signaling.

We transfected a fluorescent reporter of PKA activity, AKAR2 [2], into neonatal rat cardiac myocytes. AKAR2 is a recombinant protein containing two GFP mutants (CFP and YFP), a PKA substrate, and a phosphotyrosine-binding domain FHA. In AKAR2-krho, the membrane-targeting domain of the signaling protein Rho has been fused to AKAR2. When expressed in neonatal cardiac myocytes, AKAR2 and AKAR2-krho exhibited distinct localization patterns (see FIG 1).

We used AKAR2 and AKAR2-krho to measure the activation of PKA in response to three agonists known increase cyclic AMP: isoproterenol, forskolin, and prostaglandin E1 (PGE1) (see FIG 2). Both cytosolic and membrane PKA activities increased rapidly and robustly to isoproterenol, with the AKAR2-krho signal responding somewhat sooner. Compared with the isoproterenol response, forskolin responses were greatly delayed, potentially due to the time required to diffuse through the plasma membrane. PGE1 increased AKAR2 but not AKAR2-krho signal, suggesting that PGE1 may not effectively activate PKA localized to the plasma membrane. This is consistent with data showing PGE1-mediated increases in cyclic AMP but not the expected downstream consequences such as increased calcium currents or contractility [1].

But does AKAR expression perturb the cell's physiology? To address this question, we included AKAR into a previously described computational model of the PKA signaling network [3]. The model predicted that very high expression of AKAR2 may delay phosphorylation of both AKAR2 and endogeneous PKA substrates, while steady-state cell behavior and AKAR2's dynamic range are remarkably insensitive. We validated these predictions by comparing AKAR responses in multiple neighboring HEK293 cells with varying AKAR expression, using initial fluorescence as a relative measure of AKAR expression. For the observed range of expression, AKAR caused little change in signaling properties compared with intrinsic cellular variability. When used together in this fashion, 
dynamic live-cell data can be used to validate and iteratively improve computational models, while the models aid in the interpretation of multivariate data.

\section{References}

[1] S.F. Steinberg, L.L. Brunton, Annu. Rev. Pharmacol. Toxicol, 41 (2000) 751.

[2] J. Zhang et al., Proc. Natl. Acad. Sci. USA, 98 (2001) 14997.

[3] J.J. Saucerman et al., J. Biol. Chem, 278 (2003) 47997.

[4] This research was supported by the Whitaker Foundation and the NIH through the National Biomedical Computation Resource (P41 RR08605) .
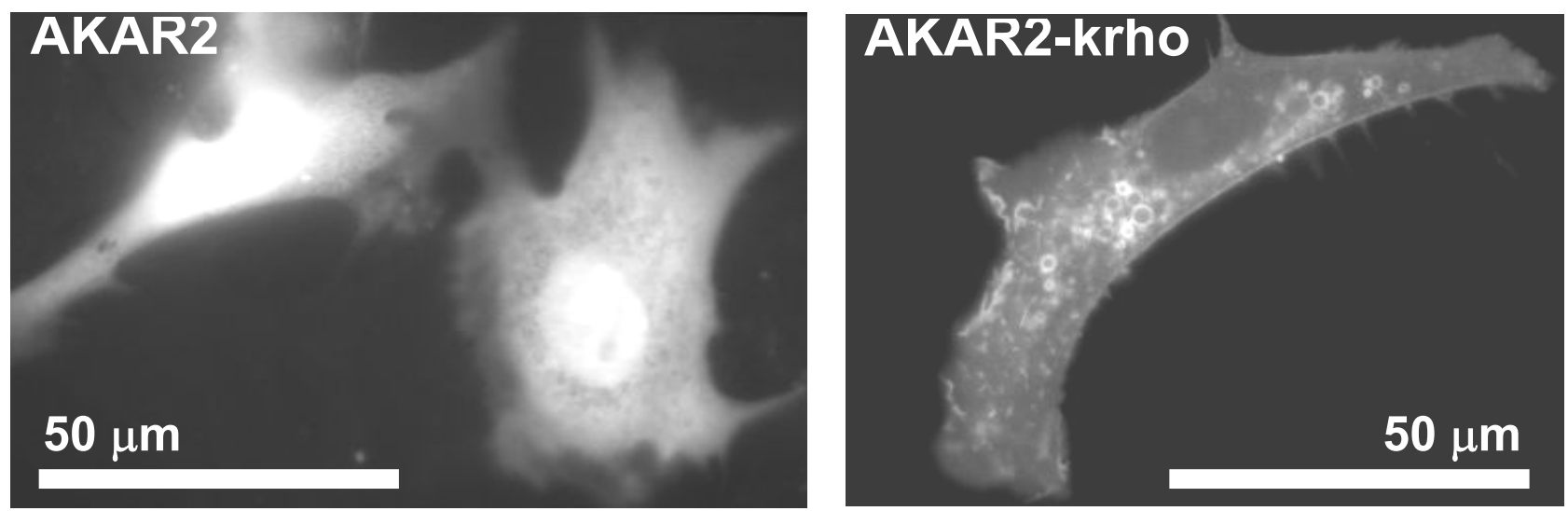

FIG. 1. Neonatal cardiac myocytes transiently transfected with either AKAR2 (left), or AKAR2krho. AKAR2 is distributed throughout the cell, while AKAR2-krho is targeted to membranes due to its krho targeting domain.

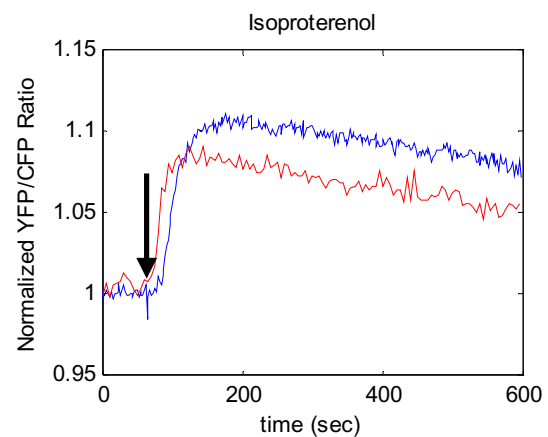

AKAR2
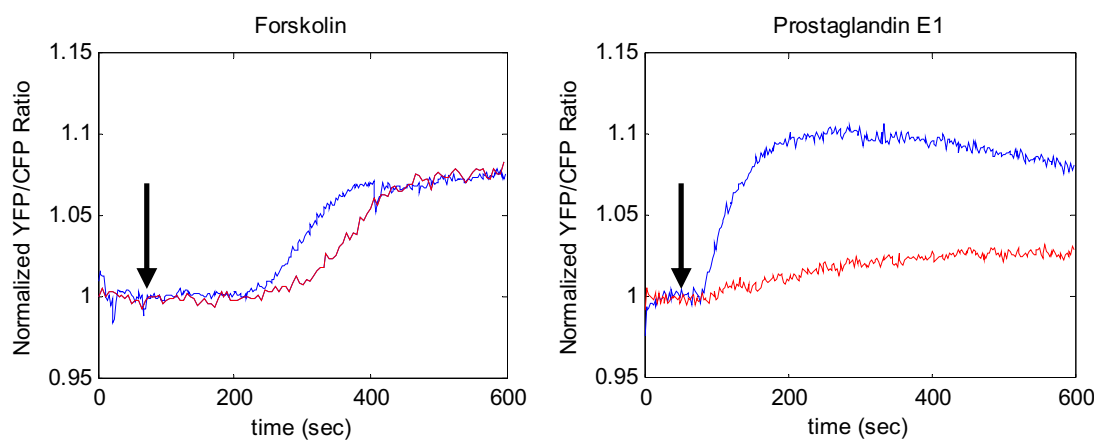

AKAR2-krho (membrane-targeted)

FIG. 2. Protein kinase A activity in live cardiac myocytes measured by AKAR2 or AKAR2-krho in response to isoproterenol (left), forskolin (middle), or prostaglandin E1 (right). The black arrow designates the time at which agonist was applied. Observation of AKAR kinetics reveals distinct modes of action for each of these agonists. 